\title{
Search for Lepton-Flavor Violating Decays $B^{+} \rightarrow K^{+} \mu^{ \pm} e^{\mp}$
}

\author{
R. Aaij et al. \\ (LHCb Collaboration)
}

(Received 4 September 2019; published 12 December 2019)

\begin{abstract}
A search for the lepton-flavor violating decays $B^{+} \rightarrow K^{+} \mu^{ \pm} e^{\mp}$ is performed using a sample of protonproton collision data, collected with the LHCb experiment at center-of-mass energies of 7 and $8 \mathrm{TeV}$ and corresponding to an integrated luminosity of $3 \mathrm{fb}^{-1}$. No significant signal is observed, and upper limits on the branching fractions are set as $\mathcal{B}\left(B^{+} \rightarrow K^{+} \mu^{-} e^{+}\right)<7.0(9.5) \times 10^{-9}$ and $\mathcal{B}\left(B^{+} \rightarrow K^{+} \mu^{+} e^{-}\right)<$ $6.4(8.8) \times 10^{-9}$ at $90 \%(95) \%$ confidence level. The results improve the current best limits on these decays by more than one order of magnitude.
\end{abstract}

DOI: 10.1103/PhysRevLett.123.241802

The observation of neutrino oscillations [1-3] has provided the first evidence for lepton-flavor violation (LFV) in neutral leptons. By contrast, LFV in the charged sector is negligible in the standard model (SM) [4] and any observation of a charged LFV decay would be indisputable evidence for physics beyond the SM (BSM). In light of recent flavor anomalies in semileptonic $b \rightarrow s \ell^{+} \ell^{-}$transitions [5-7], many SM extensions have been proposed that link leptonuniversality violation to LFV [8], predicting in particular a significantly enhanced decay rate in $b \rightarrow s \mu^{\mp} e^{ \pm}$processes. In this Letter, a search for the decays of $B^{+} \rightarrow K^{+} \mu^{ \pm} e^{\mp}$ is reported (inclusion of charge-conjugate processes is implied throughout the Letter). Their branching fractions are predicted to be in the range $10^{-8}-10^{-10}$ [9] in leptoquark models $[10,11]$, extended gauge boson models [12], or models including $C P$ violation in the neutrino sector [13]. Currently, the best limits of $\mathcal{B}\left(B^{+} \rightarrow K^{+} \mu^{-} e^{+}\right)<9.1 \times 10^{-8}$ and $\mathcal{B}\left(B^{+} \rightarrow K^{+} \mu^{+} e^{-}\right)<13 \times 10^{-8}$ have been set by the $B A B A R$ Collaboration at the $90 \%$ confidence level [14].

A dataset of proton-proton ( $p p)$ collisions at the Large Hadron Collider (LHC) corresponding to an integrated luminosity of $3 \mathrm{fb}^{-1}$, recorded with the LHCb detector in 2011 and 2012 at center-of-mass energies of 7 and $8 \mathrm{TeV}$, respectively, is used in this analysis. The two final states with different lepton charge combinations are studied independently, since they could be affected differently by BSM dynamics. The yields of the $B^{+} \rightarrow K^{+} \mu^{ \pm} e^{\mp}$ decays are normalized to those of the $B^{+} \rightarrow K^{+} J / \psi\left(\rightarrow \mu^{+} \mu^{-}\right)$ decay, which has a well-known branching fraction [15], the same topology, and similar signatures in the detector.

*Full author list given at the end of the article.

Published by the American Physical Society under the terms of the Creative Commons Attribution 4.0 International license. Further distribution of this work must maintain attribution to the author(s) and the published article's title, journal citation, and DOI. Funded by SCOAP ${ }^{3}$.
The $B^{+} \rightarrow K^{+} J / \psi\left(\rightarrow e^{+} e^{-}\right)$decay is also used as a control channel in the analysis.

The $\mathrm{LHCb}$ detector is a single-arm forward spectrometer covering the pseudorapidity range $2<\eta<5$, described in detail in Refs. $[16,17]$. The detector includes a siliconstrip vertex detector surrounding the $p p$ interaction region, tracking stations located on either side of a dipole magnet, ring-imaging Cherenkov (RICH) detectors, calorimeters, and muon chambers.

The online event selection is performed by a trigger [18], which consists of a hardware stage, based on information from the calorimeter and muon systems, followed by a software stage, which applies a full event reconstruction. At the hardware trigger stage, $B^{+} \rightarrow K^{+} \mu^{ \pm} e^{\mp}$ and $B^{+} \rightarrow K^{+} J /$ $\psi\left(\rightarrow \mu^{+} \mu^{-}\right)$event candidates are required to have a muon with high transverse momentum with respect to the beam axis, $p_{T}$. In the subsequent software trigger, at least one charged particle must have a $p_{T}>1.7 \mathrm{GeV} / c$ in the 2011 dataset and $p_{T}>1.6 \mathrm{GeV} / c$ in 2012 , unless the particle is identified as a muon, in which case $p_{T}>1.0 \mathrm{GeV} / c$ is required. This track must be significantly displaced from any primary interaction vertex (PV) in the event. Finally, a twoor three-track secondary vertex with a significant displacement from any PV is required, where a multivariate algorithm [19] is used for the identification of secondary vertices consistent with the weak decay of a $b$ hadron.

Simulated samples are used to evaluate signal efficiencies, to train multivariate classifiers, to model the shape of the invariant mass of the $B^{+} \rightarrow K^{+} \mu^{ \pm} e^{\mp}$ signal candidates, and to study physics backgrounds. In the simulation, $p p$ collisions are generated using PYTHIA $[20,21]$ with a specific LHCb configuration [22]. Decays of unstable particles are described by EVTGEN [23], in which finalstate radiation is generated using РнOTOS [24]. A phasespace model is adopted for signal $B^{+} \rightarrow K^{+} \mu^{ \pm} e^{\mp}$ decays. The interaction of the generated particles with the detector, and its response, are implemented using the GEANT4 toolkit [25] as described in Ref. [26]. 
The $B^{+} \rightarrow K^{+} \mu^{ \pm} e^{\mp}$ candidates passing the trigger selection are reconstructed by combining three charged tracks originating from a good-quality common vertex. The tracks forming the $B^{+}$candidate are required not to originate from any PV and must have sizable transverse momentum. Because of the long lifetime of the $B^{+}$meson, this vertex is required to be well separated from any $\mathrm{PV}$. The $B^{+}$direction vector, determined from its production and decay vertex positions, must be aligned with its momentum vector. The mass of the reconstructed $B^{+}$candidate, $m\left(K^{+} \mu^{ \pm} e^{\mp}\right)$, is restricted to lie within $\pm 1500 \mathrm{MeV} / c^{2}$ of the known $B^{+}$meson mass [15]. Furthermore, the $B^{+}$-meson decay products must be well identified as a kaon, an electron, and a muon, exploiting information from the Cherenkov detectors, the calorimeters, and the muon stations. The electron candidate kinematics are corrected for bremsstrahlung photon emission if a compatible photon candidate in the calorimeter is found. Kaon and electron candidates that have hits in the muon stations consistent with their trajectories are rejected. The same selection is applied to the normalization (control) channels, for which the dimuon (dielectron) invariant mass is additionally required to be consistent with the known $J / \psi$ mass [15]. The selection and analysis procedures were developed without inspecting the signal data in the region $m\left(K^{+} \mu^{ \pm} e^{\mp}\right) \in[4985,5385] \mathrm{MeV} / c^{2}$.

The most significant backgrounds originate from partially reconstructed $B^{+}$decays, e.g., from double semileptonic $B^{+} \rightarrow \bar{D}^{0} X \ell^{+} \nu_{\ell}$ with $\bar{D}^{0} \rightarrow K^{+} Y \ell^{\prime-} \bar{\nu}_{\ell^{\prime}}$ decays, where $X$ and $Y$ represent hadrons, while $\ell$ and $\ell^{\prime}$ are leptons. They are removed by imposing the requirement $m\left(K^{+} \ell^{-}\right)>$ $1885 \mathrm{MeV} / c^{2}$. Contributions from decays involving charmonium resonances, where one lepton is misidentified as a kaon or as a lepton of a different flavor, are rejected by mass vetoes.

The combinatorial background, which consists of random tracks that are associated with a common vertex, is reduced using a boosted decision tree (BDT) [27,28] algorithm. This BDT combines information about the $B^{+}$meson kinematics and information related to its flight distance, decay vertex quality, and impact parameter with respect to the primary vertex. In addition, it uses information such as the impact parameters of the electron, muon, and kaon candidates, and the isolation of the $B^{+}$candidate from any other charged track in the event [29].

The BDT is trained on simulated $B^{+} \rightarrow K^{+} \mu^{ \pm} e^{\mp}$ events that have satisfied the previous requirements. The simulated samples are adjusted using $B^{+} \rightarrow K^{+} J / \psi\left(\rightarrow \mu^{+} \mu^{-}\right)$ and $B^{+} \rightarrow K^{+} J / \psi\left(\rightarrow e^{+} e^{-}\right)$decays in data to correct data-simulation differences in the $B$-meson production kinematics, vertex quality, and detector occupancy represented by the number of tracks in the detector. The upper-mass sideband, corresponding to $m\left(K^{+} \mu^{ \pm} e^{\mp}\right) \in$ $[5385,6000] \mathrm{MeV} / c^{2}$, is used as a proxy for the background. The training is performed using a $k$-folding approach [30] with ten folds, which allows the whole sample to be used without biasing the output of the classifier. The optimal requirement on the BDT classifier is chosen to give the best expected upper limits on the branching fractions $\mathcal{B}\left(B^{+} \rightarrow K^{+} \mu^{ \pm} e^{\mp}\right)$.

The candidates surviving this multivariate selection are used to train a second BDT, dedicated to reject background from partially reconstructed $b$-hadron decays. The background sample for the training is taken from the lowermass sideband in data, corresponding to $m\left(K^{+} \mu^{ \pm} e^{\mp}\right) \in$ $[4550,4985] \mathrm{MeV} / c^{2}$, where the partially reconstructed background is expected to contribute. The signal proxy is the same as for the first BDT. The training procedure shares the $k$-folding approach and the same set of discriminating variables used to construct the first multivariate discriminant, with the addition of the ratio between the projections of the electron and the $K^{+} \mu^{ \pm}$momenta orthogonal to the $B$-meson direction of flight. The requirements on the second BDT are optimized in the same manner as the first BDT. The final stage of the selection, where requirements on the particle identification (PID) variables based on a neural net classifier for the kaon, electron, and muon are applied [31], ensures the suppression of candidates from decays with misidentification of at least one particle.

The performance of the PID algorithms is not perfectly simulated, and thus a correction is performed using highpurity calibration data samples of muons from $B \rightarrow X J /$ $\psi\left(\rightarrow \mu^{+} \mu^{-}\right)$decays, electrons from $B^{+} \rightarrow K^{+} J / \psi\left(\rightarrow e^{+} e^{-}\right)$ decays, and kaons from $D^{*+} \rightarrow D^{0}\left(\rightarrow K^{-} \pi^{+}\right) \pi^{+}$decays [32]. The calibration data are binned in the particle's momentum and pseudorapidity, and in the detector occupancy. Particle identification variables for the simulated datasets are sampled from the distributions of calibration data in the corresponding bin. The performance of the PID resampling is validated on both the $B^{+} \rightarrow K^{+} J / \psi\left(\rightarrow \mu^{+} \mu^{-}\right)$ and $B^{+} \rightarrow K^{+} J / \psi\left(\rightarrow e^{+} e^{-}\right)$control channels.

The potential contamination from $b$-hadron decays in the signal mass region after selection is analyzed using dedicated simulated samples. Two categories are analyzed: fully reconstructed $B$ decays, with at least one particle in the final state misidentified, such as the semileptonic decays $B^{+} \rightarrow K^{+} \ell^{+} \ell^{-}$and $B^{+} \rightarrow K^{+} J / \psi\left(\rightarrow \ell^{+} \ell^{-}\right)$, or fully hadronic $B^{+}$decays as $B^{+} \rightarrow K^{+} \pi^{+} \pi^{-}$; partially reconstructed decays in which at least one particle is not reconstructed and one or more particles are misidentified in addition, such as $B^{0} \rightarrow K^{* 0} \ell^{+} \ell^{-}, \Lambda_{b}^{0} \rightarrow p K^{-} \ell^{+} \ell^{-}$, $\Lambda_{b}^{0} \rightarrow p K^{-} J / \psi\left(\rightarrow \ell^{+} \ell^{-}\right)$, and $B^{+} \rightarrow \bar{D}^{0} \ell^{+} \nu_{\ell}$ transitions, where the $\bar{D}^{0}$ meson decays further to $K^{+} \pi^{-}$or $K^{+} \ell^{-} \bar{\nu}_{\ell}$. The expected number of candidates from each possible background source after the selection is evaluated from simulation and is found to be negligible.

The branching fraction $\mathcal{B}\left(B^{+} \rightarrow K^{+} \mu^{ \pm} e^{\mp}\right)$ is measured relative to the normalization channel using 


$$
\begin{aligned}
\mathcal{B}\left(B^{+} \rightarrow K^{+} \mu^{ \pm} e^{\mp}\right) & =N\left(B^{+} \rightarrow K^{+} \mu^{ \pm} e^{\mp}\right) \times \alpha, \\
\alpha & \equiv \frac{\mathcal{B}\left[B^{+} \rightarrow K^{+} J / \psi\left(\rightarrow \mu^{+} \mu^{-}\right)\right]}{\varepsilon\left(B^{+} \rightarrow K^{+} \mu^{ \pm} e^{\mp}\right)} \frac{\varepsilon\left[B^{+} \rightarrow K^{+} J / \psi\left(\rightarrow \mu^{+} \mu^{-}\right)\right]}{N\left[B^{+} \rightarrow K^{+} J / \psi\left(\rightarrow \mu^{+} \mu^{-}\right)\right]},
\end{aligned}
$$

where the $\varepsilon\left[B^{+} \rightarrow K^{+} J / \psi\left(\rightarrow \mu^{+} \mu^{-}\right)\right]$and $\varepsilon\left(B^{+} \rightarrow K^{+} \mu^{ \pm} e^{\mp}\right)$ denote the efficiencies of the normalization and signal channels, respectively; $N\left[B^{+} \rightarrow K^{+} J / \psi\left(\rightarrow \mu^{+} \mu^{-}\right)\right]$and $N\left(B^{+} \rightarrow K^{+} \mu^{ \pm} e^{\mp}\right) \quad$ are the observed $B^{+} \rightarrow K^{+} J /$ $\psi\left(\rightarrow \mu^{+} \mu^{-}\right)$and $B^{+} \rightarrow K^{+} \mu^{ \pm} e^{\mp}$ yields, respectively. The value of the branching fraction of the normalization mode is $\mathcal{B}\left[B^{+} \rightarrow K^{+} J / \psi\left(\rightarrow \mu^{+} \mu^{-}\right)\right]=(6.02 \pm 0.17) \times 10^{-5}$, taken from Ref. [15]. The yield of the normalization channel is determined from an unbinned extended maximumlikelihood fit to the invariant mass $m\left(K^{+} \mu^{+} \mu^{-}\right)$of the selected $B^{+} \rightarrow K^{+} J / \psi\left(\rightarrow \mu^{+} \mu^{-}\right)$candidates, performed separately on 2011 and 2012 data. The sum of two Crystal Ball functions [33] is used to parameterize the signal, while an exponential function models the background. The yields resulting from the fits are $26940 \pm 170$ for 2011 and $59220 \pm 250$ for 2012 data.

The efficiencies are calculated taking into account all selection requirements. The analysis is performed assuming a phase-space model for the signal decay. Efficiency maps in bins of the invariant masses of the particles in the final state $m_{K^{+} e^{ \pm}}^{2}$ and $m_{K^{+} \mu^{\mp}}^{2}$ are provided in Fig. 1 to allow for the interpretation of the result in different BSM scenarios.

All efficiencies are determined from calibrated simulation samples and the normalization factors for the two decay channels are given in Table I. The two data taking periods are combined into a single normalization factor taking into account the relative data sizes and efficiencies. The ratio $\alpha / \mathcal{B}\left[B^{+} \rightarrow K^{+} J / \psi\left(\rightarrow \mu^{+} \mu^{-}\right)\right]$, which excludes external inputs, is also quoted.

The invariant-mass distribution of $B^{+} \rightarrow K^{+} \mu^{ \pm} e^{\mp}$ candidates is modeled differently depending on whether bremsstrahlung photons have been included in the momentum calculation for the electrons. The sum of two Crystal

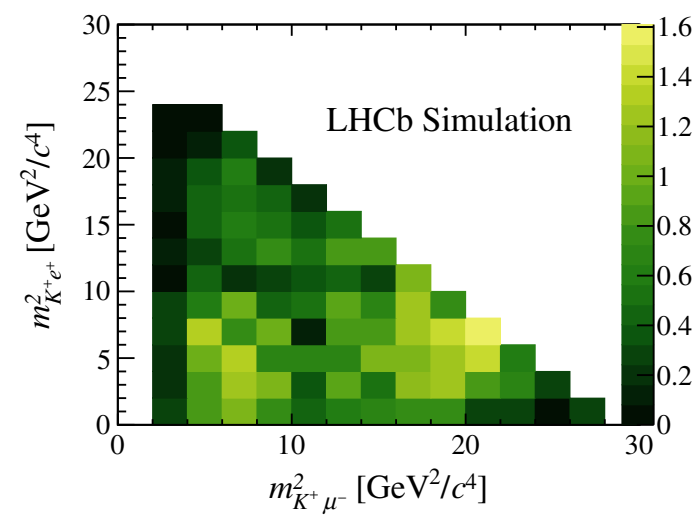

Ball functions with common mean value is used in both cases. For candidates in which bremsstrahlung photons have been included the tails are on opposite sides of the peak. Otherwise, the two tails share the same parameters. Their values, obtained from the $B^{+} \rightarrow K^{+} \mu^{ \pm} e^{\mp}$ simulation, are corrected taking into account the differences between data and simulation in $B^{+} \rightarrow K^{+} J / \psi\left(\rightarrow \mu^{+} \mu^{-}\right)$and $B^{+} \rightarrow$ $K^{+} J / \psi\left(\rightarrow e^{+} e^{-}\right)$decays. Two types of unbinned maximum-likelihood fits are performed on the dataset. The first fit assumes only background is present, with the background modeled with an exponential function. From this fit $3.9 \pm 1.1$ and $0.9 \pm 0.6$ background candidates are expected in the signal mass window for the $B^{+} \rightarrow K^{+} \mu^{+} e^{-}$and $B^{+} \rightarrow K^{+} \mu^{-} e^{+}$modes, respectively. The second fit includes the signal component, which is used to determine the signal yields. The $B^{+} \rightarrow K^{+} \mu^{-} e^{+}$and $B^{+} \rightarrow K^{+} \mu^{+} e^{-}$invariantmass distributions are fitted separately.

After unblinding the dataset, there are 1 (2) candidates in the signal mass window $m\left(K^{+} \mu^{ \pm} e^{\mp}\right) \in[5100$, 5370] $\mathrm{MeV} / c^{2}$ for the $B^{+} \rightarrow K^{+} \mu^{-} e^{+}\left(B^{+} \rightarrow K^{+} \mu^{+} e^{-}\right)$ channels, respectively, in agreement with the backgroundonly hypothesis (cf. Fig. 2). The upper limits on the branching fractions are set with the $\mathrm{CL}_{s}$ method [34], using the GammaCombo framework [35,36] with a onesided test statistic. The likelihoods are computed from fits to the invariant-mass distributions with the normalization constant constrained to its nominal value accounting for statistical and systematic uncertainties. Pseudoexperiments, in which the nuisance parameters are input at their best fit value and the background yield is varied according to its systematic uncertainty, are used for the evaluation of the test statistic. The resulting upper limits are shown in Fig. 3 and Table II.

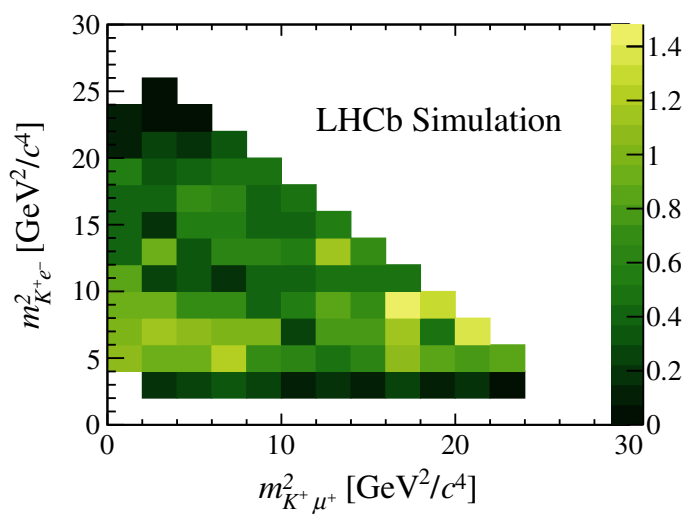

FIG. 1. Efficiency of (left) $B^{+} \rightarrow K^{+} \mu^{-} e^{+}$and (right) $B^{+} \rightarrow K^{+} \mu^{+} e^{-}$as a function of the squared invariant masses $m_{K^{+}}^{2} e^{ \pm}$and $m_{K^{+} \mu^{\mp}}^{2}$. The variation of efficiency across the Dalitz plane is due to applied vetoes. The efficiencies are given in per mille. 
TABLE I. Normalization factor $\alpha$ for $B^{+} \rightarrow K^{+} \mu^{-} e^{+}$and $B^{+} \rightarrow K^{+} \mu^{+} e^{-}$final states. The ratio $\alpha / \mathcal{B}\left[B^{+} \rightarrow K^{+} J /\right.$ $\left.\psi\left(\rightarrow \mu^{+} \mu^{-}\right)\right]$is independent of external inputs.

\begin{tabular}{lcc}
\hline \hline & $\alpha$ & $\alpha / \mathcal{B}\left[B^{+} \rightarrow K^{+} J /\right.$ \\
& $\left.\alpha\left(\rightarrow \mu^{+} \mu^{-}\right)\right]$ \\
\hline$B^{+} \rightarrow K^{+} \mu^{-} e^{+}$ & $(1.97 \pm 0.14) \times 10^{-9}$ & $(3.27 \pm 0.22) \times 10^{-5}$ \\
$B^{+} \rightarrow K^{+} \mu^{+} e^{-}$ & $(2.21 \pm 0.14) \times 10^{-9}$ & $(3.68 \pm 0.22) \times 10^{-5}$ \\
\hline \hline
\end{tabular}

The dominant sources of systematic uncertainty on the upper limits are due to applied simulation corrections. These include the kinematic difference of $B$-meson production, residual difference between correcting the muon and electron candidates, and PID resampling. Furthermore, the determination of trigger efficiencies and the knowledge of the background invariant-mass distribution are also considered in evaluating the systematic uncertainty.

The systematic uncertainty on the sampling procedure of the PID variables includes two components. The first stems from applying the sPlot [37] method to the calibration data, and adds an uncertainty of $0.1 \%$ for kaons and muons, and $3 \%$ for electrons, the latter being a conservative estimate originating from a comparison of the sPlot method with a cut-and-count method. The second component addresses the choice of binning in the sampling procedure. It is evaluated by recalculating the normalization factor $\alpha$ using a finer and a coarser binning, and taking the largest deviation with respect to the baseline result.

A small difference in the correction procedure is observed depending on the choice of control channel, namely, $B^{+} \rightarrow$ $K^{+} J / \psi\left(\rightarrow \mu^{+} \mu^{-}\right)$or $B^{+} \rightarrow K^{+} J / \psi\left(\rightarrow e^{+} e^{-}\right)$. This difference, referred to as electron-muon difference, is taken as systematic uncertainty.

The systematic uncertainty from the fitting model is determined to be $2.1 \%$ using a bootstrapping approach. The systematic uncertainty on the background model is calculated by repeating the fit using an alternative model, where the exponential function is obtained from a sample enriched in background events. The difference between the alternative and nominal background parametrization is taken as a systematic uncertainty. The uncertainty on the knowledge
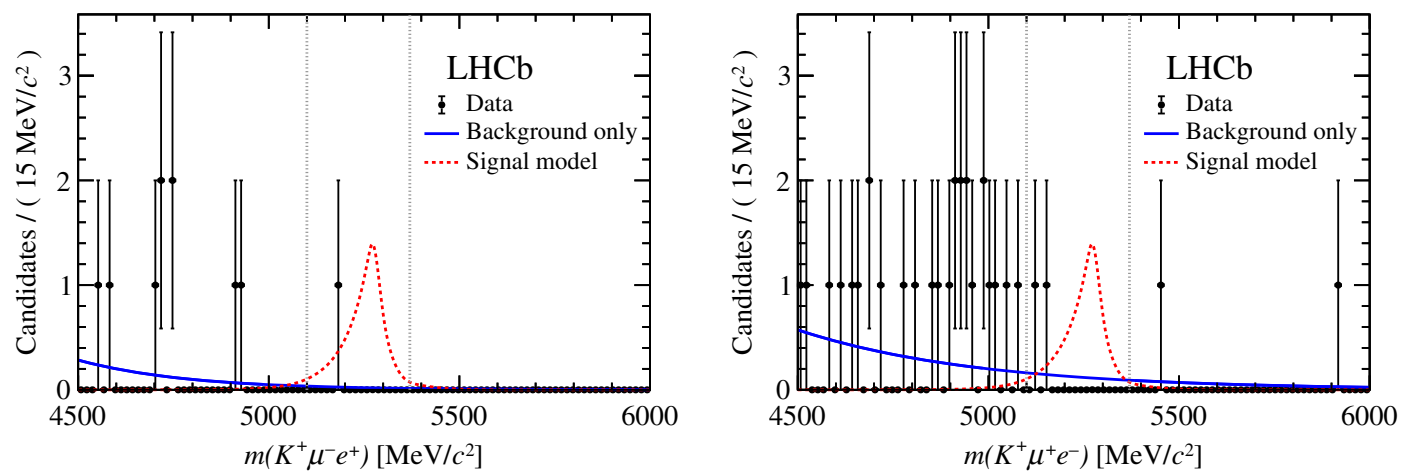

FIG. 2. Invariant-mass distributions of the (left) $B^{+} \rightarrow K^{+} \mu^{-} e^{+}$and (right) $B^{+} \rightarrow K^{+} \mu^{+} e^{-}$candidates obtained on the combined datasets recorded in 2011 and 2012 with background only fit functions (blue continuous line) and the signal model normalized to 10 candidates (red dashed line) superimposed. The signal window is indicated with gray dotted lines. Difference between the two distributions arises from the effect of the $m\left(K^{+} \ell^{-}\right)$requirement.
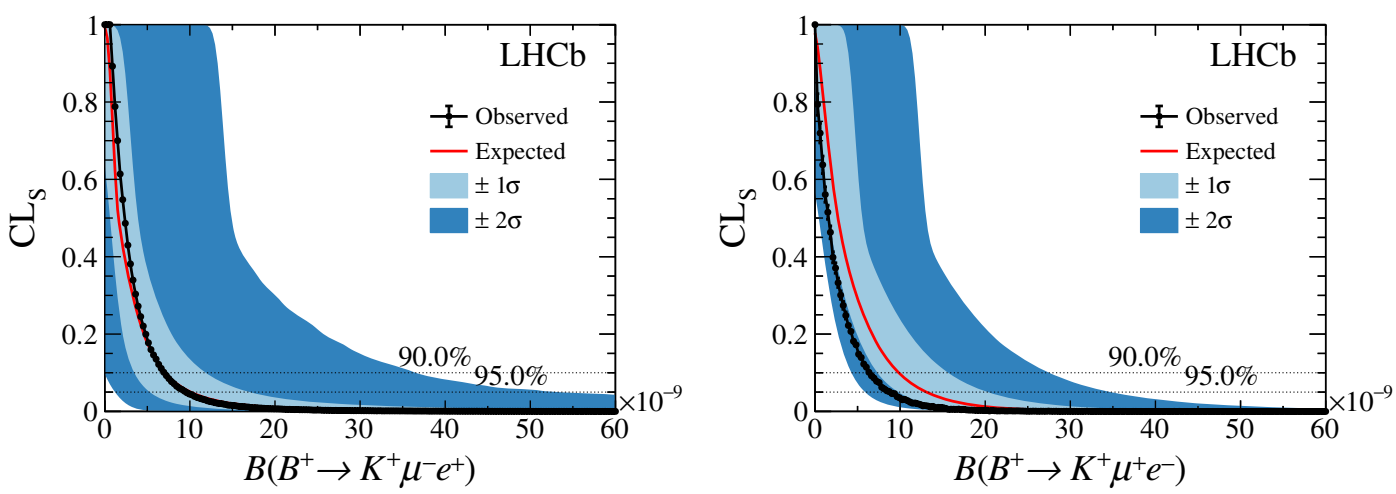

FIG. 3. Upper limits on the branching fractions of (left) $B^{+} \rightarrow K^{+} \mu^{-} e^{+}$and (right) $B^{+} \rightarrow K^{+} \mu^{+} e^{-}$decays obtained on the combined datasets recorded in 2011 and 2012. The red solid line (black solid line with data points) corresponds to the distribution of the expected (observed) upper limits, and the light blue (dark blue) band contains the $1 \sigma(2 \sigma)$ uncertainties. 
TABLE II. Upper limit on the branching fraction of $B^{+} \rightarrow$ $K^{+} \mu^{-} e^{+}$and $B^{+} \rightarrow K^{+} \mu^{+} e^{-}$decays obtained on the combined datasets recorded in 2011 and 2012 for confidence levels of $90 \%$ and $95 \%$.

\begin{tabular}{lcc}
\hline \hline & $90 \%$ C.L. & $95 \%$ C.L. \\
\hline $\mathcal{B}\left(B^{+} \rightarrow K^{+} \mu^{-} e^{+}\right) / 10^{-9}$ & 7.0 & 9.5 \\
$\mathcal{B}\left(B^{+} \rightarrow K^{+} \mu^{+} e^{-}\right) / 10^{-9}$ & 6.4 & 8.8 \\
\hline \hline
\end{tabular}

TABLE III. Summary of systematic uncertainties.

\begin{tabular}{lcc}
\hline \hline Effect & $B^{+} \rightarrow K^{+} \mu^{+} e^{-}$ & $B^{+} \rightarrow K^{+} \mu^{-} e^{+}$ \\
\hline Data-simulation corrections & $1.0 \%$ & $1.0 \%$ \\
Electron-muon differences & $1.4 \%$ & $1.4 \%$ \\
Fitting model & $2.1 \%$ & $2.1 \%$ \\
PID resampling & $4.5 \%$ & $5.5 \%$ \\
Trigger & $1.0 \%$ & $1.0 \%$ \\
Normalization factor & $3.5 \%$ & $3.5 \%$ \\
Total & $6.4 \%$ & $7.1 \%$ \\
Background & 0.60 & 0.43 \\
\hline \hline
\end{tabular}

of the $B^{+} \rightarrow K^{+} J / \psi\left(\rightarrow \mu^{+} \mu^{-}\right)$branching fraction is combined with the uncertainty due to the limited size of the simulation sample and is propagated to the normalization constant, corresponding to a systematic uncertainty of $3.5 \%$. A summary of systematic uncertainties is reported in Table III.

In conclusion, a search for the lepton-flavor violating decays $B^{+} \rightarrow K^{+} \mu^{ \pm} e^{\mp}$ is performed using data from proton-proton collisions recorded with the $\mathrm{LHCb}$ experiment at center-of-mass energies of 7 and $8 \mathrm{Tev}$, corresponding to an integrated luminosity of $3 \mathrm{fb}^{-1}$. A uniform distribution of signal events within the phase space accessible to the final-state particles is assumed. No excess is observed over the background-only hypothesis, and the resulting upper limits on the branching fractions are $\mathcal{B}\left(B^{+} \rightarrow K^{+} \mu^{-} e^{+}\right)<7.0(9.5) \times 10^{-9}$ and $\mathcal{B}\left(B^{+} \rightarrow\right.$ $\left.K^{+} \mu^{+} e^{-}\right)<6.4(8.8) \times 10^{-9}$ at $90 \%$ (95)\% confidence level. The results improve the current best limits on the decays [14] by more than 1 order of magnitude. Moreover, the measurements impose strong constraints on the aforementioned extensions to the SM [10-13].

We express our gratitude to our colleagues in the CERN accelerator departments for the excellent performance of the LHC. We thank the technical and administrative staff at the LHCb institutes. We acknowledge support from CERN and from the national agencies: CAPES, CNPq, FAPERJ, and FINEP (Brazil); MOST and NSFC (China); CNRS/ IN2P3 (France); BMBF, DFG, and MPG (Germany); INFN (Italy); NWO (Netherlands); MNiSW and NCN (Poland); MEN/IFA (Romania); MSHE (Russia); MinECo (Spain); SNSF and SER (Switzerland); NASU (Ukraine); STFC
(United Kingdom); DOE NP and NSF (USA). We acknowledge the computing resources that are provided by CERN, IN2P3 (France), KIT, and DESY (Germany), INFN (Italy), SURF (Netherlands), PIC (Spain), GridPP (United Kingdom), RRCKI and Yandex LLC (Russia), CSCS (Switzerland), IFIN-HH (Romania), CBPF (Brazil), PL-GRID (Poland) and OSC (USA). We are indebted to the communities behind the multiple open-source software packages on which we depend. Individual groups or members have received support from AvH Foundation (Germany); EPLANET, Marie Skłodowska-Curie Actions, and ERC (European Union); ANR, Labex P2IO, and OCEVU, and Région Auvergne-Rhône-Alpes (France); Key Research Program of Frontier Sciences of CAS, CAS PIFI, and the Thousand Talents Program (China); RFBR, RSF, and Yandex LLC (Russia); GVA, XuntaGal, and GENCAT (Spain); the Royal Society and the Leverhulme Trust (United Kingdom).

[1] Y. Fukuda et al. (Super-Kamiokande Collaboration), Evidence for Oscillation of Atmospheric Neutrinos, Phys. Rev. Lett. 81, 1562 (1998).

[2] Q. R. Ahmad et al. (SNO Collaboration), Measurement of the Rate of $\nu_{e}+d \rightarrow p+p+e^{-}$Interactions Produced by ${ }^{8} B$ Solar Neutrinos at the Sudbury Neutrino Observatory, Phys. Rev. Lett. 87, 071301 (2001).

[3] Q. R. Ahmad et al. (SNO Collaboration), Direct Evidence for Neutrino Flavor Transformation from Neutral Current Interactions in the Sudbury Neutrino Observatory, Phys. Rev. Lett. 89, 011301 (2002).

[4] M. Raidal et al., Flavour physics of leptons and dipole moments, Eur. Phys. J. C 57, 13 (2008).

[5] R. Aaij et al. (LHCb Collaboration), Search for LeptonUniversality Violation in $B^{+} \rightarrow K^{+} \ell^{+} \ell^{-}$Decays, Phys. Rev. Lett. 122, 191801 (2019).

[6] R. Aaij et al. (LHCb Collaboration), Test of lepton universality with $B^{0} \rightarrow K^{* 0} \ell^{+} \ell^{-}$decays, J. High Energy Phys. 08 (2017) 055.

[7] R. Aaij et al. (LHCb Collaboration), Angular analysis of the $B^{0} \rightarrow K^{* 0} \mu^{+} \mu^{-}$decay using $3 \mathrm{fb}^{-1}$ of integrated luminosity, J. High Energy Phys. 02 (2016) 104.

[8] S. L. Glashow, D. Guadagnoli, and K. Lane, Lepton Flavor Violation in B Decays?, Phys. Rev. Lett. 114, 091801 (2015).

[9] D. Guadagnoli and K. Lane, Charged-lepton mixing and lepton flavor violation, Phys. Lett. B 751, 54 (2015).

[10] I. de Medeiros Varzielas and G. Hiller, Clues for flavor from rare lepton and quark decays, J. High Energy Phys. 06 (2015) 072.

[11] G. Hiller, D. Loose, and K. Schönwald, Leptoquark flavor patterns \& B decay anomalies, J. High Energy Phys. 12 (2016) 027.

[12] A. Crivellin, L. Hofer, J. Matias, U. Nierste, S. Pokorski, and J. Rosiek, Lepton-flavour violating $B$ decays in generic $Z^{\prime}$ models, Phys. Rev. D 92, 054013 (2015). 
[13] S. M. Boucenna, J. W. F. Valle, and A. Vicente, Are the $B$ decay anomalies related to neutrino oscillations?, Phys. Lett. B 750, 367 (2015).

[14] B. Aubert et al. (BABAR Collaboration), Measurements of branching fractions, rate asymmetries, and angular distributions in the rare decays $B \rightarrow K \ell^{+} \ell^{-}$and $B \rightarrow K^{*} \ell^{+} \ell^{-}$, Phys. Rev. D 73, 092001 (2006).

[15] M. Tanabashi et al. (Particle Data Group), Review of particle physics, Phys. Rev. D 98, 030001 (2018).

[16] A. A. Alves Jr. et al. (LHCb Collaboration), The LHCb detector at the LHC, J. Instrum. 3, S08005 (2008).

[17] R. Aaij et al. (LHCb Collaboration), LHCb detector performance, Int. J. Mod. Phys. A 30, 1530022 (2015).

[18] R. Aaij et al., The LHCb trigger and its performance in 2011, J. Instrum. 8, P04022 (2013).

[19] V. V. Gligorov and M. Williams, Efficient, reliable and fast high-level triggering using a bonsai boosted decision tree, J. Instrum. 8, P02013 (2013).

[20] T. Sjöstrand, S. Mrenna, and P. Skands, A brief introduction to PYTHIA8.1, Comput. Phys. Commun. 178, 852 (2008).

[21] T. Sjöstrand, S. Mrenna, and P. Skands, PYTHIA6.4 physics and manual, J. High Energy Phys. 05 (2006) 026.

[22] I. Belyaev et al., Handling of the generation of primary events in Gauss, the LHCb simulation framework, J. Phys. 331, 032047 (2011).

[23] D. J. Lange, The EVTGEN particle decay simulation package, Nucl. Instrum. Methods Phys. Res., Sect. A 462, 152 (2001).

[24] P. Golonka and Z. Was, Рнотоs Monte Carlo: A precision tool for QED corrections in $Z$ and $W$ decays, Eur. Phys. J. C 45, 97 (2006).

[25] J. Allison, K. Amako, J. Apostolakis, H. Araujo, P. A. Dubois et al. (GEANT4 Collaboration), GEANT4 developments and applications, IEEE Trans. Nucl. Sci. 53, 270 (2006); S. Agostinelli et al. (GEANT4 Collaboration), GEANT4: A simulation toolkit, Nucl. Instrum. Methods Phys. Res., Sect. A 506, 250 (2003).
[26] M. Clemencic et al., The LHCb simulation application, Gauss: Design, evolution and experience, J. Phys. 331, 032023 (2011).

[27] L. Breiman, J. H. Friedman, R. A. Olshen, and C. J. Stone, Classification and Regression Trees (Wadsworth international group, Belmont, California, USA, 1984).

[28] Y. Freund and R. E. Schapire, A decision-theoretic generalization of on-line learning and an application to boosting, J. Comput. Syst. Sci. 55, 119 (1997).

[29] T. Aaltonen et al. (CDF Collaboration), Search for $B_{s}^{0} \rightarrow$ $\mu^{+} \mu^{-}$and $B_{d}^{0} \rightarrow \mu^{+} \mu^{-}$Decays with $2 \mathrm{fb}^{-1}$ of $p \bar{p}$ Collisions, Phys. Rev. Lett. 100, 101802 (2008).

[30] A. Bevan, R. G. Goñi, T. Stevenson, and T. Stevenson, Support vector machines and generalisation in HEP, J. Phys. 898, 072021 (2017).

[31] R. Aaij et al., Selection and processing of calibration samples to measure the particle identification performance of the LHCb experiment in Run 2, EPJ Tech. Instrum. 6, 1 (2018).

[32] L. Anderlini et al., The PIDCalib package, Report No. LHCb-PUB-2016-021.

[33] T. Skwarnicki, A study of the radiative cascade transitions between the Upsilon-prime and Upsilon resonances, Ph.D. thesis, Institute of Nuclear Physics, Krakow, 1986 [Report No. DESY-F31-86-02].

[34] A. L. Read, Presentation of search results: The $\mathrm{CL}_{\mathrm{s}}$ technique, J. Phys. G 28, 2693 (2002).

[35] R. Aaij et al. (LHCb Collaboration), Measurement of the CKM angle $\gamma$ from a combination of LHCb results, J. High Energy Phys. 12 (2016) 087.

[36] M. Kenzie, M. Karbach, T. Mombächer, M. Schlupp, and K. Schubert, GammaCombo framework for combinations of measurements and computation of confidence intervals: Public Release v1.1, https://doi.org/10.5281/zenodo .3373613 (2019).

[37] M. Pivk and F. R. Le Diberder, sPlot: A statistical tool to unfold data distributions, Nucl. Instrum. Methods Phys. Res., Sect. A 555, 356 (2005).

R. Aaij, ${ }^{28}$ C. Abellán Beteta, ${ }^{46}$ T. Ackernley, ${ }^{56}$ B. Adeva,${ }^{43}$ M. Adinolfi,${ }^{50}$ C. A. Aidala, ${ }^{77}$ Z. Ajaltouni, ${ }^{6}$ S. Akar,${ }^{61}$ P. Albicocco, ${ }^{19}$ J. Albrecht, ${ }^{11}$ F. Alessio, ${ }^{44}$ M. Alexander ${ }^{55}$ A. Alfonso Albero, ${ }^{42}$ G. Alkhazov, ${ }^{34}$ P. Alvarez Cartelle, ${ }^{57}$ A. A. Alves Jr., ${ }^{43}$ S. Amato, ${ }^{2}$ Y. Amhis, ${ }^{8}$ L. An, ${ }^{18}$ L. Anderlini, ${ }^{18}$ G. Andreassi, ${ }^{45}$ M. Andreotti, ${ }^{17}$ J. E. Andrews, ${ }^{62}$ F. Archilli, ${ }^{13}$ P. d'Argent, ${ }^{13}$ J. Arnau Romeu, ${ }^{7}$ A. Artamonov, ${ }^{41}$ M. Artuso, ${ }^{63}$ K. Arzymatov, ${ }^{38}$ E. Aslanides ${ }^{7}$ M. Atzeni, ${ }^{46}$ B. Audurier, ${ }^{23}$ S. Bachmann, ${ }^{13}$ J. J. Back, ${ }^{52}$ S. Baker, ${ }^{57}$ V. Balagura, ${ }^{8, \mathrm{c}}$ W. Baldini, ${ }^{17,44}$ A. Baranov ${ }^{38}$ R. J. Barlow, ${ }^{58}$ S. Barsuk, ${ }^{8}$ W. Barter,${ }^{57}$ M. Bartolini, ${ }^{20, i}$ F. Baryshnikov, ${ }^{74}$ V. Batozskaya, ${ }^{32}$ B. Batsukh,${ }^{63}$ A. Battig, ${ }^{11}$ V. Battista, ${ }^{45}$ A. Bay, ${ }^{45}$ M. Becker, ${ }^{11}$ F. Bedeschi, ${ }^{25}$ I. Bediaga, ${ }^{1}$ A. Beiter,${ }^{63}$ L. J. Bel, ${ }^{28}$ V. Belavin, ${ }^{38}$ S. Belin, ${ }^{23}$ N. Beliy, ${ }^{66}$ V. Bellee, ${ }^{45}$ K. Belous ${ }^{41}$ I. Belyaev ${ }^{35}$ E. Ben-Haim, ${ }^{9}$ G. Bencivenni, ${ }^{19}$ S. Benson, ${ }^{28}$ S. Beranek, ${ }^{10}$ A. Berezhnoy,${ }^{36}$ R. Bernet ${ }^{46}$ D. Berninghoff, ${ }^{13}$ E. Bertholet, ${ }^{9}$ A. Bertolin, ${ }^{24}$ C. Betancourt, ${ }^{46}$ F. Betti, ${ }^{16, f}$ M. O. Bettler, ${ }^{51}$ M. van Beuzekom, ${ }^{28}$ Ia. Bezshyiko, ${ }^{46}$ S. Bhasin, ${ }^{50}$ J. Bhom, ${ }^{30}$ M. S. Bieker, ${ }^{11}$ S. Bifani, ${ }^{49}$ P. Billoir, ${ }^{9}$ A. Birnkraut, ${ }^{11}$ A. Bizzeti, ${ }^{18, v}$ M. Bjørn, ${ }^{59}$ M. P. Blago, ${ }^{44}$ T. Blake, ${ }^{52}$ F. Blanc, ${ }^{45}$ S. Blusk, ${ }^{63}$ D. Bobulska, ${ }^{55}$ V. Bocci, ${ }^{27}$ O. Boente Garcia, ${ }^{43}$ T. Boettcher, ${ }^{60}$ A. Boldyrev, ${ }^{39}$ A. Bondar, ${ }^{40, y}$ N. Bondar, ${ }^{34}$ S. Borghi,${ }^{58,44}$ M. Borisyak, ${ }^{38}$ M. Borsato, ${ }^{13}$ J. T. Borsuk, ${ }^{30}$ M. Boubdir, ${ }^{10}$ T. J. V. Bowcock, ${ }^{56}$ C. Bozzi, ${ }^{17,44}$ S. Braun, ${ }^{13}$ A. Brea Rodriguez, ${ }^{43}$ M. Brodski, ${ }^{44}$ J. Brodzicka, ${ }^{30}$ A. Brossa Gonzalo, ${ }^{52}$ D. Brundu, ${ }^{23,44}$ E. Buchanan, ${ }^{50}$ A. Buonaura, ${ }^{46}$ C. Burr, ${ }^{58}$ A. Bursche, ${ }^{23}$ J. S. Butter, ${ }^{28}$ J. Buytaert, ${ }^{44}$ W. Byczynski, ${ }^{44}$ S. Cadeddu, ${ }^{23}$ H. Cai, ${ }^{68}$ R. Calabrese,,${ }^{17, h}$ S. Cali,${ }^{19}$ R. Calladine, ${ }^{49}$ M. Calvi,${ }^{21, j}$ M. Calvo Gomez, ${ }^{42, \mathrm{n}}$ A. Camboni, ${ }^{42, \mathrm{n}}$ 
P. Campana, ${ }^{19}$ D. H. Campora Perez ${ }^{44}$ L. Capriotti, ${ }^{16, \mathrm{f}}$ A. Carbone,${ }^{16, f}$ G. Carboni,${ }^{26}$ R. Cardinale,${ }^{20, \mathrm{i}}$ A. Cardini,${ }^{23}$

P. Carniti, ${ }^{21, j}$ K. Carvalho Akiba, ${ }^{28}$ A. Casais Vidal, ${ }^{43}$ G. Casse, ${ }^{56}$ M. Cattaneo, ${ }^{44}$ G. Cavallero, ${ }^{20}$ R. Cenci, ${ }^{25, q}$ M. G. Chapman,${ }^{50}$ M. Charles,${ }^{9,44} \mathrm{Ph}$. Charpentier, ${ }^{44}$ G. Chatzikonstantinidis, ${ }^{49}$ M. Chefdeville, ${ }^{5}$ V. Chekalina,${ }^{38}$ C. Chen, ${ }^{3}$ S. Chen, ${ }^{23}$ A. Chernov, ${ }^{30}$ S.-G. Chitic, ${ }^{44}$ V. Chobanova, ${ }^{43}$ M. Chrzaszcz, ${ }^{44}$ A. Chubykin, ${ }^{34}$ P. Ciambrone, ${ }^{19}$ M. F. Cicala, ${ }^{52}$ X. Cid Vidal, ${ }^{43}$ G. Ciezarek, ${ }^{44}$ F. Cindolo, ${ }^{16}$ P. E. L. Clarke, ${ }^{54}$ M. Clemencic, ${ }^{44}$ H. V. Cliff, ${ }^{51}$ J. Closier, ${ }^{44}$ J. L. Cobbledick, ${ }^{58}$ V. Coco, ${ }^{44}$ J. A. B. Coelho, ${ }^{8}$ J. Cogan, ${ }^{7}$ E. Cogneras, ${ }^{6}$ L. Cojocariu, ${ }^{33}$ P. Collins,${ }^{44}$ T. Colombo, ${ }^{44}$ A. Comerma-Montells, ${ }^{13}$ A. Contu, ${ }^{23}$ N. Cooke, ${ }^{49}$ G. Coombs, ${ }^{55}$ S. Coquereau, ${ }^{42}$ G. Corti, ${ }^{44}$ C. M. Costa Sobral,${ }^{52}$ B. Couturier, ${ }^{44}$ G. A. Cowan, ${ }^{54}$ D. C. Craik ${ }^{60}$ A. Crocombe,${ }^{52}$ M. Cruz Torres, ${ }^{1}$ R. Currie,${ }^{54}$ C. D’Ambrosio, ${ }^{44}$ C. L. Da Silva, ${ }^{78}$ E. Dall'Occo, ${ }^{28}$ J. Dalseno, ${ }^{43,50}$ A. Danilina, ${ }^{35}$ A. Davis, ${ }^{58}$ O. De Aguiar Francisco, ${ }^{44}$ K. De Bruyn, ${ }^{44}$ S. De Capua,${ }^{58}$ M. De Cian, ${ }^{45}$ J. M. De Miranda, ${ }^{1}$ L. De Paula, ${ }^{2}$ M. De Serio, ${ }^{15, \mathrm{e}}$ P. De Simone, ${ }^{19}$ C. T. Dean,${ }^{78}$ W. Dean,${ }^{77}$ D. Decamp,${ }^{5}$ L. Del Buono, ${ }^{9}$ B. Delaney ${ }^{51}$ H.-P. Dembinski, ${ }^{12}$ M. Demmer, ${ }^{11}$ A. Dendek,${ }^{31}$ V. Denysenko, ${ }^{46}$ D. Derkach, ${ }^{39}$ O. Deschamps, ${ }^{6}$ F. Desse, ${ }^{8}$ F. Dettori ${ }^{23}$ B. Dey, ${ }^{69}$ A. Di Canto, ${ }^{44}$ P. Di Nezza, ${ }^{19}$ S. Didenko, ${ }^{74}$ H. Dijkstra, ${ }^{44}$ F. Dordei, ${ }^{23}$ M. Dorigo, ${ }^{25, z}$

A. Dosil Suárez, ${ }^{43}$ L. Douglas, ${ }^{55}$ A. Dovbnya,${ }^{47}$ K. Dreimanis, ${ }^{56}$ M. W. Dudek, ${ }^{30}$ L. Dufour,${ }^{44}$ G. Dujany, ${ }^{9}$ P. Durante,${ }^{44}$ J. M. Durham, ${ }^{78}$ D. Dutta, ${ }^{58}$ R. Dzhelyadin, ${ }^{41, a}$ M. Dziewiecki, ${ }^{13}$ A. Dziurda, ${ }^{30}$ A. Dzyuba, ${ }^{34}$ S. Easo, ${ }^{53}$ U. Egede,${ }^{57}$ V. Egorychev, ${ }^{35}$ S. Eidelman, ${ }^{40, y}$ S. Eisenhardt ${ }^{54}$ U. Eitschberger, ${ }^{11}$ S. Ek-In, ${ }^{45}$ R. Ekelhof, ${ }^{11}$ L. Eklund, ${ }^{55}$ S. Ely, ${ }^{63}$ A. Ene, ${ }^{33}$ S. Escher, ${ }^{10}$ S. Esen, ${ }^{28}$ T. Evans, ${ }^{61}$ A. Falabella, ${ }^{16}$ N. Farley, ${ }^{49}$ S. Farry ${ }^{56}$ D. Fazzini, ${ }^{8}$ P. Fernandez Declara, ${ }^{44}$ A. Fernandez Prieto, ${ }^{43}$ F. Ferrari, ${ }^{16, f}$ L. Ferreira Lopes, ${ }^{45}$ F. Ferreira Rodrigues, ${ }^{2}$ S. Ferreres Sole, ${ }^{28}$ M. Ferro-Luzzi, ${ }^{44}$ S. Filippov, ${ }^{37}$ R. A. Fini, ${ }^{15}$ M. Fiorini, ${ }^{17, h}$ M. Firlej, ${ }^{31}$ K. M. Fischer,${ }^{59}$ C. Fitzpatrick, ${ }^{44}$ T. Fiutowski, ${ }^{31}$ F. Fleuret,,${ }^{8, c}$ M. Fontana, ${ }^{44}$ F. Fontanelli, ${ }^{20, i}$ R. Forty, ${ }^{44}$ V. Franco Lima, ${ }^{56}$ M. Franco Sevilla, ${ }^{62}$ M. Frank,${ }^{44}$ C. Frei, ${ }^{44}$ D. A. Friday, ${ }^{55}$ J. Fu, ${ }^{22, \mathrm{r}}$ W. Funk, ${ }^{44}$ M. Féo, ${ }^{44}$ E. Gabriel,${ }^{54}$ A. Gallas Torreira, ${ }^{43}$ D. Galli, ${ }^{16, f}$ S. Gallorini, ${ }^{24}$ S. Gambetta, ${ }^{54}$ Y. Gan, ${ }^{3}$ M. Gandelman, ${ }^{2}$ P. Gandini, ${ }^{22}$ Y. Gao, ${ }^{3}$ L. M. Garcia Martin ${ }^{76}$ B. Garcia Plana, ${ }^{43}$ F. A. Garcia Rosales, ${ }^{8}$ J. García Pardiñas, ${ }^{46}$ J. Garra Tico, ${ }^{51}$ L. Garrido, ${ }^{42}$ D. Gascon,${ }^{42}$ C. Gaspar,${ }^{44}$ G. Gazzoni, ${ }^{6}$ D. Gerick, ${ }^{13}$ E. Gersabeck, ${ }^{58}$ M. Gersabeck, ${ }^{58}$ T. Gershon ${ }^{52}$ D. Gerstel, ${ }^{7}$ Ph. Ghez, ${ }^{5}$ V. Gibson, ${ }^{51}$ A. Gioventù, ${ }^{43}$ O. G. Girard, ${ }^{45}$ P. Gironella Gironell, ${ }^{42}$ L. Giubega,${ }^{33}$ K. Gizdov, ${ }^{54}$ V. V. Gligorov, ${ }^{9}$ D. Golubkov, ${ }^{35}$ A. Golutvin, ${ }^{57,74}$ A. Gomes, ${ }^{1, b}$ I. V. Gorelov, ${ }^{36}$ C. Gotti, ${ }^{21, j}$ E. Govorkova, ${ }^{28}$ J. P. Grabowski, ${ }^{13}$ R. Graciani Diaz, ${ }^{42}$ T. Grammatico, ${ }^{9}$ L. A. Granado Cardoso,${ }^{44}$ E. Graugés, ${ }^{42}$ E. Graverini, ${ }^{45}$ G. Graziani, ${ }^{18}$ A. Grecu, ${ }^{33}$ R. Greim, ${ }^{28}$ P. Griffith, ${ }^{23}$ L. Grillo, ${ }^{58}$ L. Gruber,${ }^{44}$ B. R. Gruberg Cazon, ${ }^{59}$ C. Gu, ${ }^{3}$ X. Guo, ${ }^{67}$ E. Gushchin, ${ }^{37}$ A. Guth, ${ }^{10}$ Yu. Guz ${ }^{41,44}$ T. Gys, ${ }^{44}$ C. Göbel, ${ }^{65}$ T. Hadavizadeh,${ }^{59}$ C. Hadjivasiliou, ${ }^{6}$ G. Haefeli, ${ }^{45}$ C. Haen, ${ }^{44}$ S. C. Haines ${ }^{51}$ P. M. Hamilton, ${ }^{62}$ Q. Han, ${ }^{69}$ X. Han, ${ }^{13}$ T. H. Hancock, ${ }^{59}$ S. Hansmann-Menzemer, ${ }^{13}$ N. Harnew, ${ }^{59}$ T. Harrison, ${ }^{56}$ C. Hasse, ${ }^{44}$ M. Hatch, ${ }^{44}$ J. He ${ }^{66}$ M. Hecker, ${ }^{57}$ K. Heijhoff,${ }^{28}$ K. Heinicke, ${ }^{11}$ A. Heister, ${ }^{11}$ K. Hennessy, ${ }^{56}$ L. Henry, ${ }^{76}$ E. van Herwijnen, ${ }^{44}$ J. Heuel,${ }^{10}$ M. He $\beta,{ }^{71}$ A. Hicheur,${ }^{64}$ R. Hidalgo Charman, ${ }^{58}$ D. Hill, ${ }^{59}$ M. Hilton, ${ }^{58}$ P. H. Hopchev, ${ }^{45}$ J. Hu, ${ }^{13}$ W. Hu, ${ }^{69}$ W. Huang, ${ }^{66}$ Z. C. Huard,${ }^{61}$ W. Hulsbergen,${ }^{28}$ T. Humair, ${ }^{57}$ R. J. Hunter, ${ }^{52}$ M. Hushchyn, ${ }^{39}$ D. Hutchcroft, ${ }^{56}$ D. Hynds, ${ }^{28}$ P. Ibis, ${ }^{11}$ M. Idzik,${ }^{31}$ P. Ilten, ${ }^{49}$ A. Inglessi, ${ }^{34}$ A. Inyakin, ${ }^{41}$ K. Ivshin, ${ }^{34}$ R. Jacobsson, ${ }^{44}$ S. Jakobsen, ${ }^{44}$ J. Jalocha, ${ }^{59}$ E. Jans, ${ }^{28}$ B. K. Jashal, ${ }^{76}$ A. Jawahery, ${ }^{62}$ F. Jiang, ${ }^{3}$ M. John, ${ }^{59}$ D. Johnson, ${ }^{44}$ C. R. Jones, ${ }^{51}$ B. Jost ${ }^{44}$ N. Jurik ${ }^{59}$ S. Kandybei, ${ }^{47}$ M. Karacson,${ }^{44}$ J. M. Kariuki, ${ }^{50}$ S. Karodia, ${ }^{55}$ N. Kazeev, ${ }^{39}$ M. Kecke, ${ }^{13}$ F. Keizer, ${ }^{51}$ M. Kelsey ${ }^{63}$ M. Kenzie, ${ }^{51}$ T. Ketel ${ }^{29}$ B. Khanji, ${ }^{44}$ A. Kharisova, ${ }^{75}$ C. Khurewathanakul, ${ }^{45}$ K. E. Kim, ${ }^{63}$

T. Kirn, ${ }^{10}$ V. S. Kirsebom, ${ }^{45}$ S. Klaver, ${ }^{19}$ K. Klimaszewski, ${ }^{32}$ S. Koliiev, ${ }^{48}$ M. Kolpin, ${ }^{13}$ A. Kondybayeva, ${ }^{74}$

A. Konoplyannikov, ${ }^{35}$ P. Kopciewicz, ${ }^{31}$ R. Kopecna, ${ }^{13}$ P. Koppenburg ${ }^{28}$ I. Kostiuk, ${ }^{28,48}$ O. Kot, ${ }^{48}$ S. Kotriakhova, ${ }^{34}$ M. Kozeiha, ${ }^{6}$ L. Kravchuk, ${ }^{37}$ M. Kreps,${ }^{52}$ F. Kress, ${ }^{57}$ S. Kretzschmar, ${ }^{10}$ P. Krokovny, ${ }^{40, y}$ W. Krupa, ${ }^{31}$ W. Krzemien, ${ }^{32}$ W. Kucewicz, ${ }^{30, m}$ M. Kucharczyk ${ }^{30}$ V. Kudryavtsev, ${ }^{40, y}$ H. S. Kuindersma, ${ }^{28}$ G. J. Kunde, ${ }^{78}$ A. K. Kuonen, ${ }^{45}$ T. Kvaratskheliya, ${ }^{35}$ D. Lacarrere, ${ }^{44}$ G. Lafferty, ${ }^{58}$ A. Lai, ${ }^{23}$ D. Lancierini, ${ }^{46}$ J. J. Lane,${ }^{58}$ G. Lanfranchi, ${ }^{19}$ C. Langenbruch,${ }^{10}$ T. Latham, ${ }^{52}$ F. Lazzari, ${ }^{25, w}$ C. Lazzeroni, ${ }^{49}$ R. Le Gac, ${ }^{7}$ A. Leflat,${ }^{36}$ R. Lefèvre, ${ }^{6}$ F. Lemaitre, ${ }^{44}$ O. Leroy, ${ }^{7}$ T. Lesiak, ${ }^{30}$ B. Leverington, ${ }^{13}$ H. Li, ${ }^{67}$ P.-R. Li ${ }^{66, a c}$ X. Li ${ }^{78}{ }^{7}$. Li ${ }^{4}{ }^{2}$. Li,${ }^{63}$ X. Liang, ${ }^{63}$ T. Likhomanenko, ${ }^{73}$ R. Lindner, ${ }^{44}$ P. Ling, ${ }^{67}$ F. Lionetto, ${ }^{46}$ V. Lisovskyi, ${ }^{8}$ G. Liu, ${ }^{67}$ X. Liu, ${ }^{3}$ D. Loh, ${ }^{52}$ A. Loi, ${ }^{23}$ J. Lomba Castro, ${ }^{43}$ I. Longstaff, ${ }^{55}$ J. H. Lopes, ${ }^{2}$ G. Loustau, ${ }^{46}$ G. H. Lovell, ${ }^{51}$ D. Lucchesi, ${ }^{24, p}$ M. Lucio Martinez, ${ }^{28}$ Y. Luo, ${ }^{3}$ A. Lupato, ${ }^{24}$ E. Luppi, ${ }^{17, h}$ O. Lupton, ${ }^{52}$ A. Lusiani, ${ }^{25}$ X. Lyu ${ }^{66}$ R. Ma ${ }^{67}$ F. Machefert, ${ }^{8}$ F. Maciuc ${ }^{33}$ V. Macko, ${ }^{45}$ P. Mackowiak, ${ }^{11}$ S. Maddrell-Mander,${ }^{50}$ L. R. Madhan Mohan, ${ }^{50}$ O. Maev ${ }^{34,44}$ A. Maevskiy, ${ }^{39}$ K. Maguire,${ }^{58}$ D. Maisuzenko, ${ }^{34}$ M. W. Majewski, ${ }^{31}$ S. Malde,${ }^{59}$ B. Malecki, ${ }^{44}$ A. Malinin, ${ }^{73}$ T. Maltsev, ${ }^{40, y}$ H. Malygina, ${ }^{13}$ G. Manca, ${ }^{23, g}$ G. Mancinelli, ${ }^{7}$ D. Marangotto, ${ }^{22, r}$ J. Maratas, ${ }^{6, x}$ J. F. Marchand, ${ }^{5}$ U. Marconi, ${ }^{16}$ C. Marin Benito, ${ }^{8}$ M. Marinangeli, ${ }^{45}$ P. Marino, ${ }^{45}$ J. Marks, ${ }^{13}$ P. J. Marshall, ${ }^{56}$ G. Martellotti, ${ }^{27}$ L. Martinazzoli, ${ }^{44}$ M. Martinelli, ${ }^{44,21}$ D. Martinez Santos, ${ }^{43}$ F. Martinez Vidal, ${ }^{76}$ A. Massafferri, ${ }^{1}$ 
M. Materok, ${ }^{10}$ R. Matev, ${ }^{44}$ A. Mathad ${ }^{46}$ Z. Mathe, ${ }^{44}$ V. Matiunin, ${ }^{35}$ C. Matteuzzi, ${ }^{21}$ K. R. Mattioli, ${ }^{77}$ A. Mauri, ${ }^{46}$ E. Maurice,${ }^{8, \mathrm{c}}$ B. Maurin, ${ }^{45}$ M. McCann,${ }^{57,44}$ L. Mcconnell, ${ }^{14}$ A. McNab ${ }^{58}$ R. McNulty, ${ }^{14}$ J. V. Mead,${ }^{56}$ B. Meadows, ${ }^{61}$ C. Meaux ${ }^{7}$ G. Meier, ${ }^{11}$ N. Meinert, ${ }^{71}$ D. Melnychuk, ${ }^{32}$ M. Merk,${ }^{28}$ A. Merli, ${ }^{22, r}$ E. Michielin, ${ }^{24}$ D. A. Milanes, ${ }^{70}$ E. Millard, ${ }^{52}$ M.-N. Minard, ${ }^{5}$ O. Mineev, ${ }^{35}$ L. Minzoni, ${ }^{17, h}$ S. E. Mitchell,${ }^{54}$ B. Mitreska, ${ }^{58}$ D. S. Mitzel,${ }^{13}$ A. Mogini, ${ }^{9}$ R. D. Moise ${ }^{57}$ T. Mombächer,${ }^{11}$ I. A. Monroy, ${ }^{70}$ S. Monteil, ${ }^{6}$ M. Morandin,${ }^{24}$ G. Morello, ${ }^{19}$ M. J. Morello, ${ }^{25, \text { u }}$ J. Moron,${ }^{31}$ A. B. Morris, ${ }^{7}$ A. G. Morris, ${ }^{52}$ R. Mountain,${ }^{63}$ H. Mu, ${ }^{3}$ F. Muheim, ${ }^{54}$ M. Mukherjee,${ }^{69}$ M. Mulder,${ }^{28}$ C. H. Murphy, ${ }^{59}$ D. Murray, ${ }^{58}$ A. Mödden, ${ }^{11}$ D. Müller,${ }^{44}$ J. Müller, ${ }^{11}$ K. Müller, ${ }^{46}$ V. Müller,${ }^{11}$ P. Naik, ${ }^{50}$ T. Nakada, ${ }^{45}$ R. Nandakumar, ${ }^{53}$ A. Nandi, ${ }^{59}$ T. Nanut, ${ }^{45}$ I. Nasteva, ${ }^{2}$ M. Needham,${ }^{54}$ N. Neri, ${ }^{22, r}$ S. Neubert, ${ }^{13}$ N. Neufeld, ${ }^{44}$ R. Newcombe,${ }^{57}$ T. D. Nguyen, ${ }^{45}$ C. Nguyen-Mau, ${ }^{45, o}$ E. M. Niel,${ }^{8}$ S. Nieswand,${ }^{10}$ N. Nikitin, ${ }^{36}$ N. S. Nolte, ${ }^{44}$ D. P. O'Hanlon, ${ }^{16}$ A. Oblakowska-Mucha, ${ }^{31}$ V. Obraztsov, ${ }^{41}$ S. Ogilvy, ${ }^{55}$ R. Oldeman, ${ }^{23, g}$ C. J. G. Onderwater, ${ }^{72}$ J. D. Osborn, ${ }^{77}$ A. Ossowska, ${ }^{30}$ J. M. Otalora Goicochea, ${ }^{2}$ T. Ovsiannikova, ${ }^{35}$ P. Owen, ${ }^{46}$ A. Oyanguren,${ }^{76}$ P. R. Pais, ${ }^{45}$ T. Pajero, ${ }^{25, u}$ A. Palano, ${ }^{15}$ M. Palutan, ${ }^{19}$ G. Panshin, ${ }^{75}$ A. Papanestis,${ }^{53}$ M. Pappagallo, ${ }^{54}$ L. L. Pappalardo, ${ }^{17, h}$ W. Parker, ${ }^{62}$ C. Parkes,${ }^{58,44}$ G. Passaleva, ${ }^{18,44}$ A. Pastore, ${ }^{15}$ M. Patel,${ }^{57}$ C. Patrignani,,${ }^{16, f}$ A. Pearce, ${ }^{44}$ A. Pellegrino, ${ }^{28}$ G. Penso, ${ }^{27}$ M. Pepe Altarelli, ${ }^{44}$ S. Perazzini ${ }^{16}$ D. Pereima, ${ }^{35}$ P. Perret,${ }^{6}$ L. Pescatore,${ }^{45}$ K. Petridis,${ }^{50}$ A. Petrolini, ${ }^{20, i}$ A. Petrov, ${ }^{73}$ S. Petrucci, ${ }^{54}$ M. Petruzzo,${ }^{22, r}$ B. Pietrzyk,${ }^{5}$ G. Pietrzyk, ${ }^{45}$ M. Pikies, ${ }^{30}$ M. Pili, ${ }^{59}$ D. Pinci,${ }^{27}$ J. Pinzino, ${ }^{44}$ F. Pisani, ${ }^{44}$ A. Piucci, ${ }^{13}$ V. Placinta, ${ }^{33}$ S. Playfer ${ }^{54}$ J. Plews, ${ }^{49}$ M. Plo Casasus, ${ }^{43}$ F. Polci, ${ }^{9}$ M. Poli Lener, ${ }^{19}$ M. Poliakova,${ }^{63}$ A. Poluektov, ${ }^{7}$ N. Polukhina, ${ }^{74, d}$ I. Polyakov, ${ }^{63}$ E. Polycarpo, ${ }^{2}$ G. J. Pomery ${ }^{50}$ S. Ponce, ${ }^{44}$ A. Popov ${ }^{41}$ D. Popov, ${ }^{49}$ S. Poslavskii, ${ }^{41}$ K. Prasanth, ${ }^{30}$ C. Prouve, ${ }^{43}$ V. Pugatch ${ }^{48}$ A. Puig Navarro, ${ }^{46}$ H. Pullen,${ }^{59}$ G. Punzi ${ }^{25, q}$ W. Qian, ${ }^{66}$ J. Qin, ${ }^{66}$ R. Quagliani, ${ }^{9}$ B. Quintana, ${ }^{6}$ N. V. Raab, ${ }^{14}$ B. Rachwal, ${ }^{31}$ J. H. Rademacker ${ }^{50}$ M. Rama, ${ }^{25}$ M. Ramos Pernas, ${ }^{43}$ M. S. Rangel, ${ }^{2}$ F. Ratnikov, ${ }^{38,39}$ G. Raven, ${ }^{29}$ M. Ravonel Salzgeber, ${ }^{44}$ M. Reboud, ${ }^{5}$ F. Redi, ${ }^{45}$ S. Reichert, ${ }^{11}$ A. C. dos Reis, ${ }^{1}$ F. Reiss, ${ }^{9}$ C. Remon Alepuz ${ }^{76}$ Z. Ren, ${ }^{3}$ V. Renaudin, ${ }^{59}$ S. Ricciardi,${ }^{53}$ S. Richards, ${ }^{50}$ K. Rinnert, ${ }^{56}$ P. Robbe, ${ }^{8}$ A. Robert,${ }^{9}$ A. B. Rodrigues,${ }^{45}$ E. Rodrigues,${ }^{61}$ J. A. Rodriguez Lopez,${ }^{70}$ M. Roehrken, ${ }^{44}$ S. Roiser, ${ }^{44}$ A. Rollings, ${ }^{59}$ V. Romanovskiy, ${ }^{41}$ A. Romero Vidal, ${ }^{43}$ J. D. Roth, ${ }^{77}$ M. Rotondo, ${ }^{19}$ M. S. Rudolph, ${ }^{63}$ T. Ruf, ${ }^{44}$ J. Ruiz Vidal, ${ }^{76}$ J. Ryzka, ${ }^{31}$ J. J. Saborido Silva, ${ }^{43}$ N. Sagidova,${ }^{34}$ B. Saitta, ${ }^{23, g}$ C. Sanchez Gras, ${ }^{28}$ C. Sanchez Mayordomo, ${ }^{76}$ B. Sanmartin Sedes,${ }^{43}$ R. Santacesaria, ${ }^{27}$ C. Santamarina Rios, ${ }^{43}$ M. Santimaria, ${ }^{19,44}$ E. Santovetti, ${ }^{26, k}$ G. Sarpis, ${ }^{58}$ A. Sarti, ${ }^{19,1}$ C. Satriano, ${ }^{27, t}$ A. Satta, ${ }^{26}$ M. Saur, ${ }^{66}$ D. Savrina, ${ }^{35,36}$ L. G. Scantlebury Smead, ${ }^{59}$ S. Schael, ${ }^{10}$ M. Schellenberg, ${ }^{11}$ M. Schiller, ${ }^{55}$ H. Schindler, ${ }^{44}$ M. Schmelling, ${ }^{12}$

T. Schmelzer, ${ }^{11}$ B. Schmidt, ${ }^{44}$ O. Schneider, ${ }^{45}$ A. Schopper, ${ }^{44}$ H. F. Schreiner, ${ }^{61}$ M. Schubiger, ${ }^{28}$ S. Schulte, ${ }^{45}$

M. H. Schune, ${ }^{8}$ R. Schwemmer, ${ }^{44}$ B. Sciascia, ${ }^{19}$ A. Sciubba, ${ }^{27,1}$ A. Semennikov, ${ }^{35}$ A. Sergi, ${ }^{49,44}$ N. Serra, ${ }^{46}$ J. Serrano, ${ }^{7}$ L. Sestini, ${ }^{24}$ A. Seuthe, ${ }^{11}$ P. Seyfert, ${ }^{44}$ M. Shapkin, ${ }^{41}$ T. Shears, ${ }^{56}$ L. Shekhtman, ${ }^{40, y}$ V. Shevchenko, ${ }^{73,74}$ E. Shmanin, ${ }^{74}$ J. D. Shupperd, ${ }^{63}$ B. G. Siddi, ${ }^{17}$ R. Silva Coutinho, ${ }^{46}$ L. Silva de Oliveira, ${ }^{2}$ G. Simi, ${ }^{24, p}$ S. Simone, ${ }^{15, e}$ I. Skiba, ${ }^{17}$ N. Skidmore, ${ }^{13}$ T. Skwarnicki, ${ }^{63}$ M. W. Slater, ${ }^{49}$ J. G. Smeaton, ${ }^{51}$ E. Smith, ${ }^{10}$ I. T. Smith ${ }^{54}$ M. Smith, ${ }^{57}$ M. Soares, ${ }^{16}$ L. Soares Lavra, ${ }^{1}$ M. D. Sokoloff, ${ }^{61}$ F. J. P. Soler, ${ }^{55}$ B. Souza De Paula, ${ }^{2}$ B. Spaan, ${ }^{11}$ E. Spadaro Norella, ${ }^{22, r}$ P. Spradlin, ${ }^{55}$ F. Stagni,${ }^{44}$ M. Stahl,${ }^{61}$ S. Stahl,${ }^{44}$ P. Stefko, ${ }^{45}$ S. Stefkova,${ }^{57}$ O. Steinkamp ${ }^{46}$ S. Stemmle, ${ }^{13}$ O. Stenyakin,${ }^{41}$ M. Stepanova,${ }^{34}$ H. Stevens, ${ }^{11}$ A. Stocchi, ${ }^{8}$ S. Stone, ${ }^{63}$ S. Stracka, ${ }^{25}$ M. E. Stramaglia, ${ }^{45}$ M. Straticiuc, ${ }^{33}$ U. Straumann, ${ }^{46}$ S. Strokov, ${ }^{75}$ J. Sun, ${ }^{3}$ L. Sun ${ }^{68}$ Y. Sun, ${ }^{62}$ K. Swientek, ${ }^{31}$ A. Szabelski, ${ }^{32}$ T. Szumlak, ${ }^{31}$ M. Szymanski, ${ }^{66}$ S. T'Jampens, ${ }^{5}$ S. Taneja, ${ }^{58}$ Z. Tang, ${ }^{3}$ T. Tekampe, ${ }^{11}$ G. Tellarini, ${ }^{17}$ F. Teubert ${ }^{44}$ E. Thomas, ${ }^{44}$ K. A. Thomson,${ }^{56}$ J. van Tilburg, ${ }^{28}$ M. J. Tilley, ${ }^{57}$ V. Tisserand, ${ }^{6}$ M. Tobin, ${ }^{4}$ S. Tolk, ${ }^{44}$ L. Tomassetti, ${ }^{17, h}$ D. Tonelli, ${ }^{25}$ D. Y. Tou, ${ }^{9}$ E. Tournefier, ${ }^{5}$ M. Traill, ${ }^{55}$ M. T. Tran, ${ }^{45}$ A. Trisovic, ${ }^{51}$ A. Tsaregorodtsev, ${ }^{7}$ G. Tuci, ${ }^{25,44, q}$ A. Tully, ${ }^{51}$ N. Tuning, ${ }^{28}$ A. Ukleja, ${ }^{32}$ A. Usachov, ${ }^{8}$ A. Ustyuzhanin, ${ }^{38,39}$ U. Uwer, ${ }^{13}$ A. Vagner, ${ }^{75}$ V. Vagnoni, ${ }^{16}$ A. Valassi, ${ }^{44}$ S. Valat, ${ }^{44}$ G. Valenti, ${ }^{16}$ H. Van Hecke, ${ }^{78}$ C. B. Van Hulse, ${ }^{14}$ R. Vazquez Gomez, ${ }^{44}$ P. Vazquez Regueiro, ${ }^{43}$ S. Vecchi, ${ }^{17}$ M. van Veghel, ${ }^{28}$ J. J. Velthuis, ${ }^{50}$ M. Veltri, ${ }^{18, s}$

A. Venkateswaran, ${ }^{63}$ M. Vernet, ${ }^{6}$ M. Veronesi, ${ }^{28}$ M. Vesterinen, ${ }^{52}$ J. V. Viana Barbosa, ${ }^{44}$ D. Vieira, ${ }^{66}$ M. Vieites Diaz, ${ }^{45}$ H. Viemann, ${ }^{71}$ X. Vilasis-Cardona, ${ }^{42, \mathrm{n}}$ A. Vitkovskiy, ${ }^{28}$ V. Volkov, ${ }^{36}$ A. Vollhardt, ${ }^{46}$ D. Vom Bruch, ${ }^{9}$ B. Voneki, ${ }^{44}$ A. Vorobyev, ${ }^{34}$ V. Vorobyev, ${ }^{40, y}$ N. Voropaev ${ }^{34}$ J. A. de Vries, ${ }^{28}$ C. Vázquez Sierra, ${ }^{28}$ R. Waldi,${ }^{71}$ J. Walsh ${ }^{25}$ J. Wang, ${ }^{4}$ J. Wang, ${ }^{3}$ M. Wang, ${ }^{3}$ Y. Wang, ${ }^{69}$ Z. Wang, ${ }^{46}$ D. R. Ward,${ }^{51}$ H. M. Wark, ${ }^{56}$ N. K. Watson, ${ }^{49}$ D. Websdale, ${ }^{57}$ A. Weiden, ${ }^{46}$ C. Weisser, ${ }^{60}$ B. D. C. Westhenry ${ }^{50}$ D. J. White,${ }^{58}$ M. Whitehead,${ }^{10}$ G. Wilkinson,${ }^{59}$ M. Wilkinson, ${ }^{63}$ I. Williams, ${ }^{51}$ M. R. J. Williams, ${ }^{58}$ M. Williams, ${ }^{60}$ T. Williams, ${ }^{49}$ F. F. Wilson, ${ }^{53}$ M. Winn, ${ }^{8}$ W. Wislicki, ${ }^{32}$ M. Witek, ${ }^{30}$ G. Wormser, ${ }^{8}$ S. A. Wotton, ${ }^{51}$ H. Wu, ${ }^{63}$ K. Wyllie, ${ }^{44}$ Z. Xiang, ${ }^{66}$ D. Xiao, ${ }^{69}$ Y. Xie, ${ }^{69}$ H. Xing, ${ }^{67}$ A. Xu,${ }^{3}$ L. Xu, ${ }^{3}$ M. Xu, ${ }^{69}$ Q. Xu, ${ }^{66}$ Z. Xu, ${ }^{3}$ Z. Xu, ${ }^{5}$ Z. Yang, ${ }^{3}$ Z. Yang, ${ }^{62}$ Y. Yao, ${ }^{63}$ L. E. Yeomans, ${ }^{56}$ H. Yin, ${ }^{69}$ J. Yu, ${ }^{69, a b}$ X. Yuan, ${ }^{63}$ O. Yushchenko, ${ }^{41}$ K. A. Zarebski, ${ }^{49}$ 
M. Zavertyaev, ${ }^{12, \mathrm{~d}}$ M. Zeng, ${ }^{3}$ D. Zhang, ${ }^{69}$ L. Zhang, ${ }^{3}$ S. Zhang, ${ }^{3}$ W. C. Zhang, ${ }^{3 \text { aa }}$ Y. Zhang, ${ }^{44}$ A. Zhelezov, ${ }^{13}$ Y. Zheng, ${ }^{66}$ X. Zhou, ${ }^{66}$ Y. Zhou, ${ }^{66}$ X. Zhu, ${ }^{3}$ V. Zhukov, ${ }^{10,36}$ J. B. Zonneveld, ${ }^{54}$ and S. Zucchelli ${ }^{16, f}$

\title{
(LHCb Collaboration)
}

\author{
${ }^{1}$ Centro Brasileiro de Pesquisas Físicas $(C B P F)$, Rio de Janeiro, Brazil \\ ${ }^{2}$ Universidade Federal do Rio de Janeiro (UFRJ), Rio de Janeiro, Brazil \\ ${ }^{3}$ Center for High Energy Physics, Tsinghua University, Beijing, China \\ ${ }^{4}$ Institute Of High Energy Physics (IHEP), Beijing, China \\ ${ }^{5}$ Univ. Grenoble Alpes, Univ. Savoie Mont Blanc, CNRS, IN2P3-LAPP, Annecy, France \\ ${ }^{6}$ Université Clermont Auvergne, CNRS/IN2P3, LPC, Clermont-Ferrand, France \\ ${ }^{7}$ Aix Marseille Univ, CNRS/IN2P3, CPPM, Marseille, France \\ ${ }^{8}$ LAL, Univ. Paris-Sud, CNRS/IN2P3, Université Paris-Saclay, Orsay, France \\ ${ }^{9}$ LPNHE, Sorbonne Université, Paris Diderot Sorbonne Paris Cité, CNRS/IN2P3, Paris, France \\ ${ }^{10}$ I. Physikalisches Institut, RWTH Aachen University, Aachen, Germany \\ ${ }^{11}$ Fakultät Physik, Technische Universität Dortmund, Dortmund, Germany \\ ${ }^{12}$ Max-Planck-Institut für Kernphysik (MPIK), Heidelberg, Germany \\ ${ }^{13}$ Physikalisches Institut, Ruprecht-Karls-Universität Heidelberg, Heidelberg, Germany \\ ${ }^{14}$ School of Physics, University College Dublin, Dublin, Ireland \\ ${ }^{15}$ INFN Sezione di Bari, Bari, Italy \\ ${ }^{16}$ INFN Sezione di Bologna, Bologna, Italy \\ ${ }^{17}$ INFN Sezione di Ferrara, Ferrara, Italy \\ ${ }^{18}$ INFN Sezione di Firenze, Firenze, Italy \\ ${ }^{19}$ INFN Laboratori Nazionali di Frascati, Frascati, Italy \\ ${ }^{20}$ INFN Sezione di Genova, Genova, Italy \\ ${ }^{21}$ INFN Sezione di Milano-Bicocca, Milano, Italy \\ ${ }^{22}$ INFN Sezione di Milano, Milano, Italy \\ ${ }^{23}$ INFN Sezione di Cagliari, Monserrato, Italy \\ ${ }^{24}$ INFN Sezione di Padova, Padova, Italy \\ ${ }^{25}$ INFN Sezione di Pisa, Pisa, Italy \\ ${ }^{26}$ INFN Sezione di Roma Tor Vergata, Roma, Italy \\ ${ }^{27}$ INFN Sezione di Roma La Sapienza, Roma, Italy \\ ${ }^{28}$ Nikhef National Institute for Subatomic Physics, Amsterdam, Netherlands \\ ${ }^{29}$ Nikhef National Institute for Subatomic Physics and VU University Amsterdam, Amsterdam, Netherlands \\ ${ }^{30}$ Henryk Niewodniczanski Institute of Nuclear Physics Polish Academy of Sciences, Kraków, Poland \\ ${ }^{31}$ AGH-University of Science and Technology, Faculty of Physics and Applied Computer Science, Kraków, Poland \\ ${ }^{32}$ National Center for Nuclear Research (NCBJ), Warsaw, Poland \\ ${ }^{33}$ Horia Hulubei National Institute of Physics and Nuclear Engineering, Bucharest-Magurele, Romania \\ ${ }^{34}$ Petersburg Nuclear Physics Institute NRC Kurchatov Institute (PNPI NRC KI), Gatchina, Russia \\ ${ }^{35}$ Institute of Theoretical and Experimental Physics NRC Kurchatov Institute (ITEP NRC KI), Moscow, Russia, Moscow, Russia \\ ${ }^{36}$ Institute of Nuclear Physics, Moscow State University (SINP MSU), Moscow, Russia \\ ${ }^{37}$ Institute for Nuclear Research of the Russian Academy of Sciences (INR RAS), Moscow, Russia \\ ${ }^{38}$ Yandex School of Data Analysis, Moscow, Russia \\ ${ }^{39}$ National Research University Higher School of Economics, Moscow, Russia \\ ${ }^{40}$ Budker Institute of Nuclear Physics (SB RAS), Novosibirsk, Russia \\ ${ }^{41}$ Institute for High Energy Physics NRC Kurchatov Institute (IHEP NRC KI), Protvino, Russia, Protvino, Russia \\ ${ }^{42}$ ICCUB, Universitat de Barcelona, Barcelona, Spain \\ ${ }^{43}$ Instituto Galego de Física de Altas Enerxías (IGFAE), Universidade de Santiago de Compostela, Santiago de Compostela, Spain \\ ${ }^{44}$ European Organization for Nuclear Research (CERN), Geneva, Switzerland \\ ${ }^{45}$ Institute of Physics, Ecole Polytechnique Fédérale de Lausanne (EPFL), Lausanne, Switzerland \\ ${ }^{46}$ Physik-Institut, Universität Zürich, Zürich, Switzerland \\ ${ }^{47}$ NSC Kharkiv Institute of Physics and Technology (NSC KIPT), Kharkiv, Ukraine \\ ${ }^{48}$ Institute for Nuclear Research of the National Academy of Sciences (KINR), Kyiv, Ukraine \\ ${ }^{49}$ University of Birmingham, Birmingham, United Kingdom \\ ${ }^{50}$ H.H. Wills Physics Laboratory, University of Bristol, Bristol, United Kingdom \\ ${ }^{51}$ Cavendish Laboratory, University of Cambridge, Cambridge, United Kingdom \\ ${ }^{52}$ Department of Physics, University of Warwick, Coventry, United Kingdom \\ ${ }^{53}$ STFC Rutherford Appleton Laboratory, Didcot, United Kingdom \\ ${ }^{54}$ School of Physics and Astronomy, University of Edinburgh, Edinburgh, United Kingdom
}




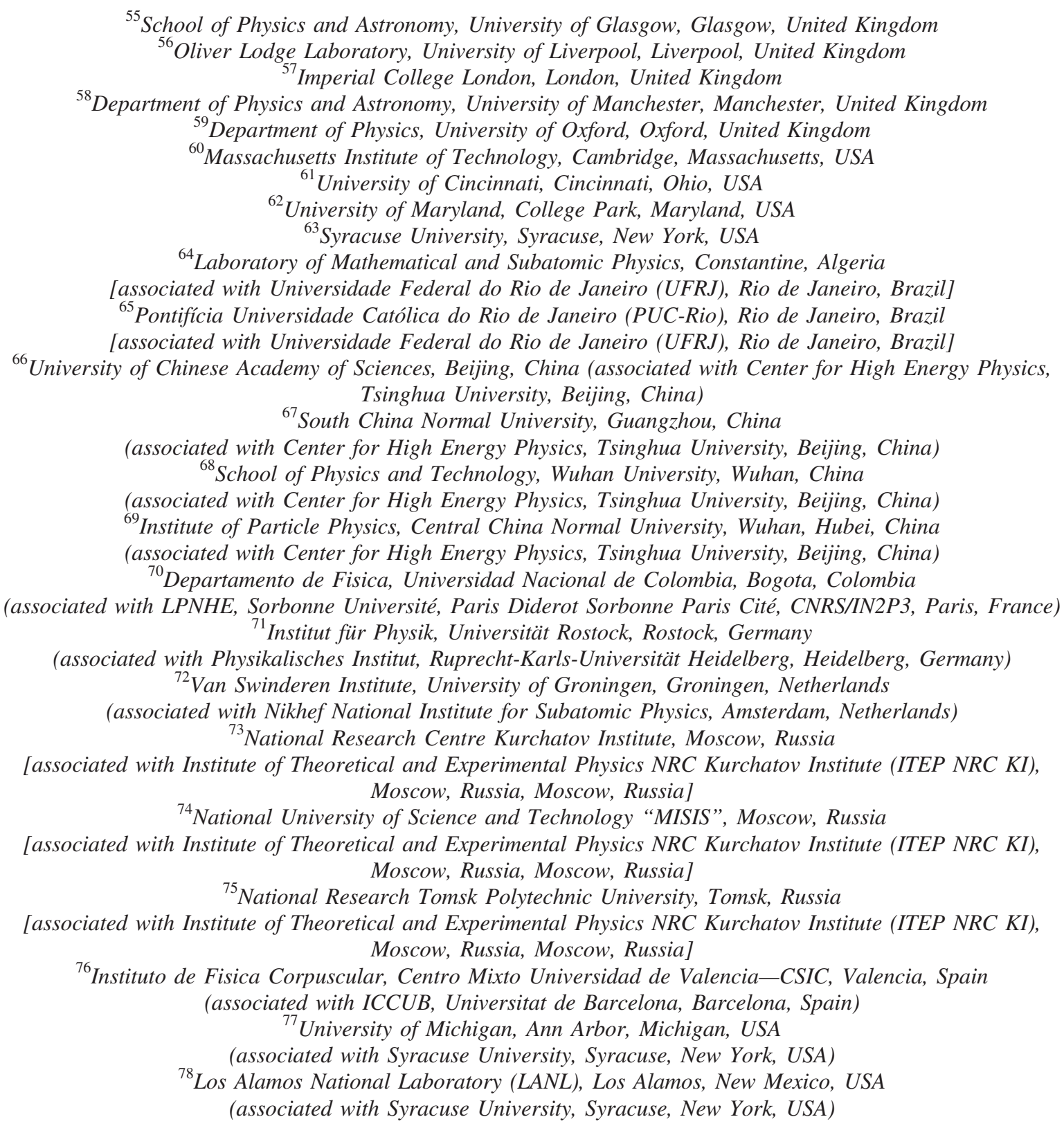

${ }^{\mathrm{a}}$ Deceased.

${ }^{\mathrm{b}}$ Also at Universidade Federal do Triângulo Mineiro (UFTM), Uberaba-MG, Brazil.

${ }^{\mathrm{c}}$ Also at Laboratoire Leprince-Ringuet, Palaiseau, France.

${ }^{\mathrm{d}}$ Also at P.N. Lebedev Physical Institute, Russian Academy of Science (LPI RAS), Moscow, Russia.

${ }^{\mathrm{e} A l s o}$ at Università di Bari, Bari, Italy.

${ }^{\mathrm{f}}$ Also at Università di Bologna, Bologna, Italy.

${ }^{\mathrm{g}}$ Also at Università di Cagliari, Cagliari, Italy.

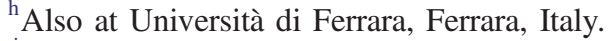

${ }^{\mathrm{i}}$ Also at Università di Genova, Genova, Italy.

${ }^{\mathrm{j}}$ Also at Università di Milano Bicocca, Milano, Italy.

${ }^{\mathrm{k}}$ Also at Università di Roma Tor Vergata, Roma, Italy.

${ }^{1}$ Also at Università di Roma La Sapienza, Roma, Italy.

${ }^{\mathrm{m}}$ Also at AGH-University of Science and Technology, Faculty of Computer Science, Electronics and Telecommunications, Kraków, Poland.

${ }^{\mathrm{n}}$ Also at LIFAELS, La Salle, Universitat Ramon Llull, Barcelona, Spain.

${ }^{\circ}$ Also at Hanoi University of Science, Hanoi, Vietnam.

${ }^{\mathrm{p}}$ Also at Università di Padova, Padova, Italy. 
${ }^{\mathrm{q}}$ Also at Università di Pisa, Pisa, Italy.

${ }^{\mathrm{r}}$ Also at Università degli Studi di Milano, Milano, Italy.

${ }^{\mathrm{s}}$ Also at Università di Urbino, Urbino, Italy.

${ }^{t}$ Also at Università della Basilicata, Potenza, Italy.

"Also at Scuola Normale Superiore, Pisa, Italy.

${ }^{v}$ Also at Università di Modena e Reggio Emilia, Modena, Italy.

${ }^{\mathrm{w}}$ Also at Università di Siena, Siena, Italy.

${ }^{\mathrm{x}}$ Also at MSU-Iligan Institute of Technology (MSU-IIT), Iligan, Philippines.

${ }^{y}$ Also at Novosibirsk State University, Novosibirsk, Russia.

${ }^{\mathrm{z}}$ Also at Sezione INFN di Trieste, Trieste, Italy.

${ }^{\text {aa }}$ Also at School of Physics and Information Technology, Shaanxi Normal University (SNNU), Xi'an, China.

${ }^{a b}$ Also at Physics and Micro Electronic College, Hunan University, Changsha City, China.

${ }^{\text {ac }}$ Also at Lanzhou University, Lanzhou, China. 